\title{
Four-Dimensional Printed Liquid Crystalline Elastomer Actuators with Fast Photoinduced Mechanical Response toward Light-Driven Robotic Functions
}

\author{
Lorena Ceamanos, Zehra Kahveci, María López-Valdeolivas, Danqing Liu, Dirk Jan Broer, \\ and Carlos Sánchez-Somolinos*
}

Cite This: ACS Appl. Mater. Interfaces 2020, 12, 44195-44204

Read Online

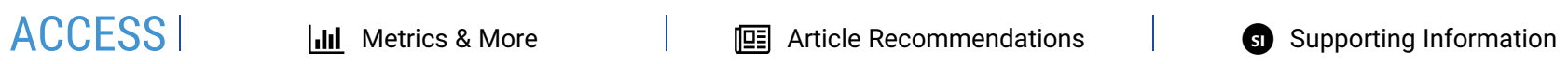

ABSTRACT: Remote light exposure of photoresponsive liquid crystalline polymers has drawn great attention over the last years as an attractive strategy to generate mechanical work with high spatial resolution. To tailor these materials into practical engineering devices, it is of key importance to gain control over their morphology and thus precisely program their mechanical response, which must also be fast and relevant in magnitude. In this communication, we report the four-dimensional (4D) printing of azobenzene-containing liquid crystalline elastomers (LCEs) that respond to light. During extrusion of the LCE precursor, mesogen orientation is defined by the needle's moving direction enabling a precise definition of the director, which is later fixed by photopolymerization. Fast mechanical responses have been observed in these 4D printed LCE elements when excited with ultraviolet (UV) light. These 4D printed elements lift objects many times heavier than their own weight, demonstrating a capacity to produce effective work. Photochemical and photothermal contributions to the deformation and force have been

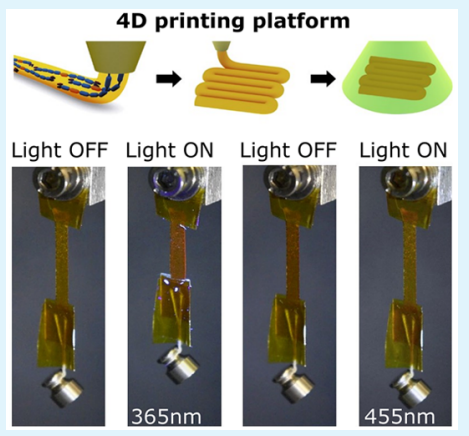
identified. Advantageously, the use of blue and UV light excitation enables adjustment of generated forces that can be maintained even in the dark and can be released by light excitation or temperature. The demonstrated ability to generate light-responsive elements quickly delivering sufficient work paves the way for implementing remotely addressed mechanical functions to future soft robotics and engineering.

KEYWORDS: 3D printing, 4D printing, photoactuators, liquid crystalline polymers, soft robotics

\section{INTRODUCTION}

Over the last years, three-dimensional (3D) printing has become a key enabling technology assisting the fast manufacturing of complex 3D objects that are difficult to be built using conventional subtractive or mold-assisted technologies. ${ }^{1-5}$ This new digital, additive fabrication paradigm enables the rapid transition of computer-aided designs (CAD) into real and complex 3D objects, shortening the development process and thus facilitating the transition of ideas into products, making a deep impact in many diverse research and application areas such as healthcare, aerospace, automotive, or robotics. ${ }^{1,2}$ Materials typically employed in 3D printing, for instance, conventional thermoplastics, usually lead to rigid and immutable objects. The concept of four-dimensional (4D) printing introduced by Tibbits adds time as a fourth dimension to generate objects that can change their shape by responding to an external stimulus. ${ }^{6}$ The capability to change shape in a programmed fashion relies on the precise deposition of the material, creating well-defined architectures and morphologies. 4D printing typically makes use of smart materials together with additive manufacturing techniques to incorporate a shape-changing ability into 3D objects in response to a stimulus. ${ }^{7-11}$ Due to their tailorability, smart responsive polymers, such as shape memory polymers $^{12-15}$ and hydrogels, ${ }^{7,8,16,17}$ are pointed as suitable candidates for 4D printing. Recently, liquid crystalline elastomers (LCEs) were introduced as promising 4D printable systems. ${ }^{18-20}$ Local anisotropy is advantageously conferred to these materials during printing. Shear forces within the needle and/or elongational flow during the deposition favor polymer chain alignment and thus orientational order of the mesogens of the main chain along the needle moving direction. ${ }^{19}$ Polymer chain alignment can later be fixed by photopolymerization leading to the LCE. It is well-known that LCEs exhibit a large anisotropic deformation as a result of a change of their state of molecular order. ${ }^{21}$ For example, an increase in the temperature results in a decrease in liquid crystalline (LC) order resulting in a contraction of the material along the preferential orientation direction and an expansion perpendicular to it. The genuine responsive character of LCEs together with the capability of

Received: July 24, 2020

Accepted: September 4, 2020

Published: September 4, 2020 
extrusion-based printing with a digital control over their molecular alignment provides an accurate programming of the magnitude and directionality of the internal stresses upon stimulation. As a result, well-defined reversible shape-morphing characteristics are prescribed to the printed elements through the digital printing process. Amine- and thiol-acrylate reactive oligomers have been demonstrated as suitable materials to create liquid crystalline elastomeric elements with well-defined morphologies. ${ }^{18-20,22,23}$ The so generated elements can change their shape in a controlled fashion over time by varying the temperature. The ability to use $3 \mathrm{D}$ printing, precisely positioning materials into complex geometries, leads to attain complex functions, far exceeding those of typically reached by thin films fabricated between glass plates with alignment layers, thus enabling the implementation of complete soft robotic elements and devices. ${ }^{19,24}$ This exciting scenario motivates us to seek for new materials presenting enhanced performance, in terms of response time, exertion of relevant forces, and response to a variety of stimuli that will allow us to deploy a broad toolbox in devising new actuator and robotic functions.

In this work, we report 4D printing of LCEs responding to light, which is selected as a remote trigger for actuation with a high degree of spatial and temporal control of excitation. By using light, no contact (pneumatic, electrical, or mechanical) with any device is required to implement and govern different deformation and mechanical functions. ${ }^{25-40}$ We have synthesized an acrylate end-capped macromer based on amine-acrylate chemistry incorporating azobenzene moieties that are wellknown molecular switches, undergoing isomerization between their trans and cis states upon illumination with appropriate wavelength. ${ }^{41}$ When integrated in LC cross-linked systems, the large geometrical change of the azobenzene moiety at the molecular level upon illumination is translated into a macroscopic mechanical response. ${ }^{31}$ Besides, photoinduced heating due to azobenzene light absorption can also contribute to the generation of mechanical stresses. A visible photoinitiator is incorporated into the formulation of the LCE to trigger its polymerization when exposed to green light, out of the absorption region of the azobenzene units. After deposition and photocuring, the response of the printed elements to temperature is evaluated. Next, the response of these same systems to ultraviolet (UV) and blue light is investigated. Irradiation with different regimes of energy led to different photomechanical responses. Unlike many other previous works in the field of liquid crystalline-based photoactuators, concentrated on studying bending phenomena, here, we have focused in studying work and force carried out by these photoresponsive elements when exposed to light as an important aspect in their application in a muscle-like configuration for soft robotic applications. Photogenerated work and force have been characterized in these $4 \mathrm{D}$ printed elements, and the mechanisms for photoactuation were discussed. Fast photomechanical response has been achieved in these systems as well as the generation of significant mechanical work when compared with temperature-responsive systems.

\section{EXPERIMENTAL SECTION}

Materials. Mesogenic diacrylate 1,4-bis-[4-(6-acryloyloxyhexyloxy)benzoyloxy]-2-methylbenzene (RM82) and the photoresponsive diacrylate, 4,4'-bis[9-(acryloyloxy)nonyloxy]azobenzene (A9A), were acquired from Synthon Chemicals GmbH. Chain extender $n$-butylamine and solvent tetrahydrofuran (THF) were purchased from
Aldrich. Photoinitiator titanium(4+) bis(2,6-difluoro-3-(1H-pyrrol-1yl)benzen-1-ide) bis(cyclopenta-2,4-dien-1-ide) (Irgacure784) was obtained from Organica Feinchemie GmbH. The sacrificial support layer to release the LCE elements was made of polyvinyl alcohol (PVA; $80 \%$ hydrolyzed; $M_{\mathrm{w}}$ of $\left.9,000-10,000\right)$ that was acquired from Aldrich.

Ink Preparation. The azobenzene-functionalized oligomer is prepared with a modification of a previously reported synthetic procedure. ${ }^{19}$ Briefly, $n$-butylamine, RM82, and A9A were added into a flask with a 1:1:0.12 molar ratio, respectively. The photoinitiator, $3.5 \mathrm{wt}$ \% Irgacure 784, was dissolved in THF, filtered with a $200 \mathrm{~nm}$ PTFE filter (VWR), and added into the mixture. Additional THF was added into the mixture to reach a 1:1 weight ratio between THF and the rest of the components. The solution was homogenized using a magnetic stirrer and allowed to react for $30 \mathrm{~min}$ at $70{ }^{\circ} \mathrm{C}$ in a closed flask. Subsequently, the flask was opened and left to stir at $70{ }^{\circ} \mathrm{C}$ for $72 \mathrm{~h}$ to evaporate THF, leading to the final photopolymerizable ink. By comparing the weight of the chemicals employed initially and that of the final ink, 1 wt \% (referred to the weight of THF initially added) has been estimated as a level of residual solvent.

3D Printing Equipment. The preparation of light-responsive elements was carried out using a homebuilt 3D printer assembled in our laboratory as previously reported. ${ }^{19}$ Basically, a computer numerical control (CNC) router chassis was coupled with a temperaturecontrolled reservoir for the ink. Pressure was applied on demand to the reservoir to extrude the ink through the needle while describing $X, Y$, and $Z$ motions. Libre-Cad freeware software was used to generate computer-aided design (CAD) files. Printing is controlled by WinPCNC software.

3D Printing of LCE Elements. The ink, loaded in a light-protected barrel, was extruded through a needle tip (23 gauge with an inner diameter of $330 \mu \mathrm{m}$ ). The printing head was heated to $60^{\circ} \mathrm{C}$, while the substrate was kept at room temperature (RT). LCE elements were printed on conventional glass microscope slides, coated with a thin layer of PVA with a thickness of $150 \mathrm{~nm}$. In order to prepare PVA-coated substrates, a 5 wt \% PVA solution was prepared with Milli-Q purified water and was deposited on a clean glass surface via spin-coating (1800 $\mathrm{rpm}$ for $60 \mathrm{~s}$ ) and left to dry at $60^{\circ} \mathrm{C}$ for $60 \mathrm{~min}$. Printing of elements was carried out on top of these PVA-coated glass substrates using the $3 \mathrm{D}$ printer, as described above. Printing speeds in the range between 6 and $12 \mathrm{~mm} / \mathrm{s}$ are typically used. Two different geometries were employed for the printed elements: a rectangular strip for bending ( 9 $\mathrm{mm} \times 3 \mathrm{~mm})$ and weight-lifting actuation $(17 \mathrm{~mm} \times 3 \mathrm{~mm})$ experiments and a dog bone-shaped sample for isometric force measurements with a test length of $15 \mathrm{~mm}$ ( $2.85 \mathrm{~mm}$ width). The director orientation of these designs, defined by the control of the printing direction, was aligned with the long axis of the sample. Photoinduced polymerization of the deposited elements was carried out by exposure to green light from a LED source (THORLABS, emission wavelength at $530 \mathrm{~nm}$, output power of $370 \mathrm{~mW}$, positioned 5 $\mathrm{cm}$ from the sample) at RT, under mild vacuum of $100 \mathrm{mbar}$, for $30 \mathrm{~min}$ each side of the printed sample. A mirror was placed beneath the samples to reflect light toward their back side and thus reinforce the photopolymerization process of the printed elements.

Characterization Techniques. Polarization Optical Microscopy. LC printed samples were examined using a polarized optical microscope (POM) (Nikon Eclipse 80i).

Thickness Characterization. Thickness measurements of the printed elements were carried out by using a profilometer, Bruker DektakXT Stylus Profiler.

Thermo- and Photoactuation Characterization. 4D printed samples were used for thermo- and photoactuation experiments. After printing and photopolymerization on the PVA-coated glass substrate, samples were immersed in water. The PVA layer dissolved within a few hours, and the LCE elements were released. After collection from water, fixation tape was attached to one extreme.

Thermoactuation experiments were performed in a homebuilt aluminum oven cavity provided with an optical access allowing the observation of the deformations. ${ }^{19}$ The samples were pinned from the sample holder on one extreme and placed inside the oven cavity. A weight of $1 \mathrm{~g}$ was attached to the extreme of the strip. Heating from RT 
to $100{ }^{\circ} \mathrm{C}$ is carried out at a rate of $5{ }^{\circ} \mathrm{C} / \mathrm{min}$. Actuation on heating was monitored by acquiring images with a digital camera Nikon D3300. Both length and width of the stripes were measured from the images using ImageJ software. Briefly, the sample height was monitored during the external stimulus by drawing in this software a straight line along the long axis of the strip, parallel to the printing direction. The boundary of the fixation tape is used as a limit for the drawn line. In addition, the sample width was monitored during the external stimulus by drawing a straight line along the short axis of the strip, perpendicular to the printing direction. The changes in length and width of the sample were characterized with normalized values divided by the initial values for each sample and experiment.

Photoactuation of these same samples was studied by exposing them to light coming from a UV LED $(365 \mathrm{~nm})$ and a blue LED $(455 \mathrm{~nm})$. Both, UV and blue light, reach the same side of the samples. For bending experiments, the sample was fixed at the upper extreme, while the other part was free to move. For weight-lifting experiments, as in the thermoactuation experiments, the samples were fixed at the upper extreme with a weight of $1 \mathrm{~g}$ attached to the lower extreme of the strip. Actuation was monitored by using images taken from a video recording, which was acquired with a digital camera Nikon D3300. The acquired images were analyzed by using a manual approach based on ImageJ as described above. The surface temperature of the films was measured using a thermal camera Gobi from Xenics.

Isometric photoinduced force measurements were carried out using a force transducer. Both ends of a dog bone-shaped LCE strip were attached to custom-made sample holders made of PMMA, leaving a test length of $15 \mathrm{~mm}$. One of the holders was fixed to a force transducer, with a measuring range of $0-2 \mathrm{~N}$, and the other one to a translation stage. Prior to light stimulation, samples are stretched to reach an initial stress by using the translation stage. Force exerted by the LCE strip was then measured upon UV stimulation with different light intensities and doses. The surface temperature of the LCE samples was also monitored in these experiments by using the thermal camera as described above.

\section{RESULTS AND DISCUSSION}

4D Printing of LCE Elements. The responsive elements studied in this work were prepared using inks comprising a reactive LC oligomer bearing acrylate end groups at the chain extremes, together with a visible light photoinitiator (Figure 1a). The LC oligomer was synthesized by the Michael addition of $n$ butylamine to two different diacrylates, RM82 and A9A, in a 1:1:0.12 molar ratio, respectively, as described in detail in the Experimental Section. The excess of acrylate groups with respect to amine ensures that the synthesized polymer chains retain the acrylate functionality at their extremes. These reactive acrylate groups can later be polymerized in a second stage by lightinitiated free-radical polymerization. RM82 does not absorb in the UV-visible region above $300 \mathrm{~nm}$, whereas the A9A diacrylate, bearing an azobenzene unit substituted at the $4^{\prime}$ position with oxygen atoms presents two absorption peaks, an intense band in the UV region and a less intense one in the blue region of the spectrum corresponding to the $\pi-\pi^{*}$ transition and $n-\pi^{*}$ transitions, respectively. ${ }^{42,43}$ The aforementioned chromophore has been thoroughly used to provide photomechanical response to LC cross-linked materials. ${ }^{42}$ LC networks bearing this chromophore in the thermodynamically stable trans form typically have a yellow-orange color, turning into dark orange upon UV irradiation due to photogeneration of cis isomers. The cis isomer has a lifetime of several hours at RT. Isomerization back from the cis to the trans state can also be induced by illumination with blue light addressing the cis isomer absorption. ${ }^{42}$ In addition, a titanocene photoinitiator, commercially known as Irgacure 784, was employed to photocure the printed samples using green light, far from the absorption region of the trans azobenzene form avoiding premature photo- a)
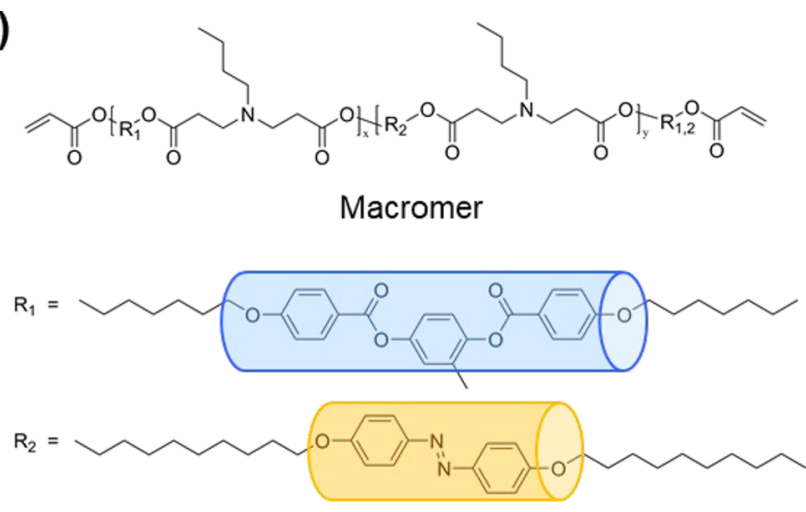

$+$

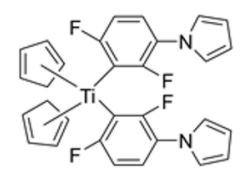

Photoinitiator

b)

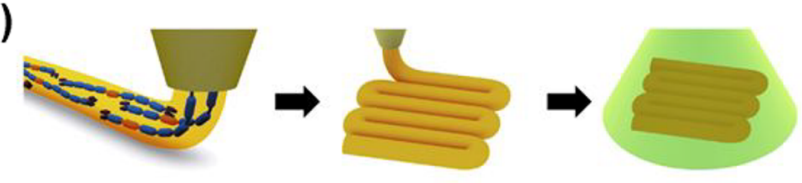

c)
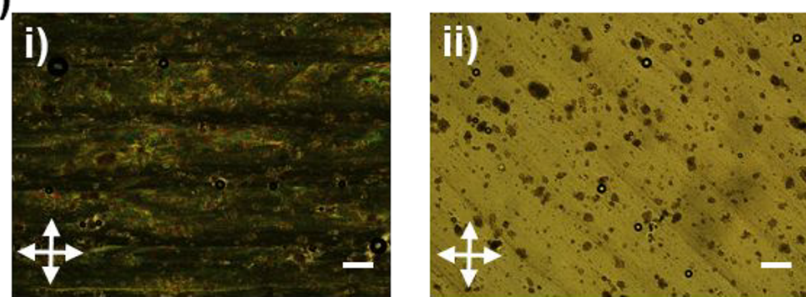

Figure 1. (a) Molecular structure of the ink components. (b) Cartoon illustration of the $4 \mathrm{D}$ printing process. Ink is digitally deposited and subsequently photopolymerized with green light. (c) POM images of closely printed lines leading to continuous strips acquired between crossed polarizers (crosses indicate polarizer transmission directions) with the printing direction oriented (i) parallel and (ii) at $45^{\circ}$ with respect to the first polarizer transmission direction (scale bar of 100 $\mu \mathrm{m})$.

isomerization effects during the photoinduced polymerization process. Ultimately, LCE elements were prepared using the printing platform developed by our group (see the Experimental Section). ${ }^{19}$ First, the ink was deposited on a PVA-coated glass substrate using an extrusion-based 3D printer with the printhead temperature preset to $60^{\circ} \mathrm{C}$. Continuous strips of LCEs with planar orientation were generated by printing closely packed parallel lines of material that fuse together. Figure 1c shows polarized optical microscope (POM) images of a continuous strip printed in this way with the printing direction at 0 and $45^{\circ}$ with regard to the transmission direction of the first polarizer. These images suggest that the monolithic uniaxial orientation of the director in the sample is imposed during the printing process. This is in accordance with previously reported studies, in which during printing, shear forces and/or elongational flow favor alignment of the polymeric chains and that of the in-chain mesogenic units that orient along the needle movement direction. ${ }^{19}$ This enables a precise digital control over the 
director field of the deposited material. Finally, the LCP precursor was turned into LCEs by photoinduced polymerization with green light. POM observations of the LCEs after curing showed that the director morphology was preserved as initially imposed to these materials during the printing step. ${ }^{19}$

Thermomechanical Response of LCE Elements. Prior to undertaking photomechanical studies with our systems, the thermomechanical response of $4 \mathrm{D}$ printed elements was evaluated. Uniaxially oriented strips with the director along the long axis of the sample were printed and cured on top of PVA-coated glass as previously described in the Experimental Section. The LCE strips, once released from the substrate, were fixed at one extreme, and a hanging weight of $1 \mathrm{~g}$ was attached to their lower end. The samples were then heated in an oven provided with an optical access for observation and thermomechanical characterization.

The first thermal cycle, heating from 30 to $100^{\circ} \mathrm{C}$ and cooling down again to $30{ }^{\circ} \mathrm{C}$, was done to erase any thermal history of the sample or residual stresses in the sample due to processing. ${ }^{21}$ This treatment was performed for all the samples in this work before actuation studies are carried out. Samples, treated this way, were subsequently subjected to thermal cycles within the same range of temperatures to assess the thermoactuation of the $4 \mathrm{D}$ printed elements. As shown in Figure 2, when the sample is
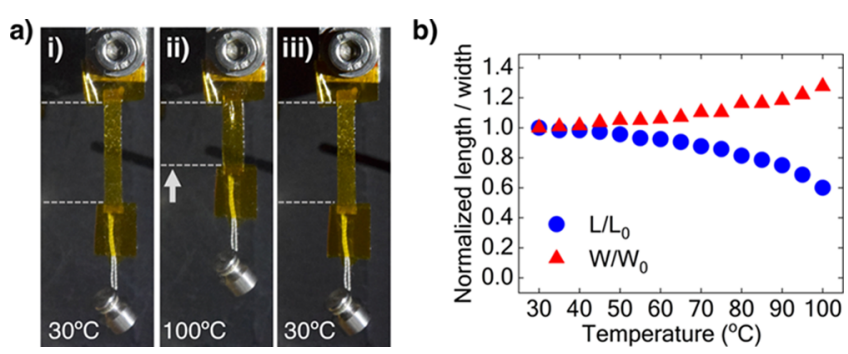

Figure 2. Thermomechanical response of a uniaxially oriented strip (90 $\mu \mathrm{m}$ thick) with the director along its long axis. (a) Weight of $1 \mathrm{~g}$ attached to the extreme of the strip lifted upon heating. (i-iii) Camera images acquired on heating from (i) $30^{\circ} \mathrm{C}$ to (ii) $100^{\circ} \mathrm{C}$ and (iii) at 30 ${ }^{\circ} \mathrm{C}$ again after cooling down. (b) Temperature dependence of normalized length and width of the LCE strip during the heating process. The strip length and width were measured at $30^{\circ} \mathrm{C}\left(L_{0}\right.$ and $W_{0}$, respectively) and during the temperature increase of the cycle ( $L$ and $W$, respectively).

heated from 30 to $100{ }^{\circ} \mathrm{C}$, a strip contraction along its long axis and subsequent weight lifting are observed. Cooling down of the sample to RT leads to elongation to its initial length (see Figure 2a-iii). A reversible behavior has been shown for samples after the first thermal cycle, indicating that the polymer network was well developed. As previously reported, the increase in mesogenic disorder, produced by the increase in temperature, causes the shortening along the director and the expansion along its perpendicular direction. ${ }^{18,19}$ Under these load conditions, the contraction along the long axis of the strip, parallel to the original printing direction, is of the order of $40 \%$ of its initial length. Simultaneously, the expansion along the short axis of the strip, perpendicular to the original printing direction, is of the order of $28 \%$ of the initial width (Figure $2 \mathrm{~b}$ ).

Photomechanical Response of LCE Elements. After assessing the thermomechanical response of the $4 \mathrm{D}$ printed elements, their photomechanical response was evaluated as a core target of this paper. Photomechanical response in azobenzene liquid crystalline cross-linked systems is typically explained on the basis of different microscopic mechanisms. On one side, illumination of azobenzene molecules in the maximum absorption band of the trans isomer leads to conversion into the cis isomer. The large geometrical change of the molecule upon isomerization induces a reduction of the orientational order degree of the liquid crystalline system. As a result, this builds up stresses that produce anisotropic deformation of the LC system with a contraction along the director and expansion perpendicular to it. ${ }^{27,41}$ On the other side, especially for high intensities and high concentrations of azobenzene, significant photoinduced heating of the sample due to light absorption can also cause a change in the orientational order degree leading to mechanical deformation. ${ }^{31}$

First, the response of free-standing films to light has been explored (Figure 3). Illumination with $50 \mathrm{~mW} / \mathrm{cm}^{2}$ of UV light
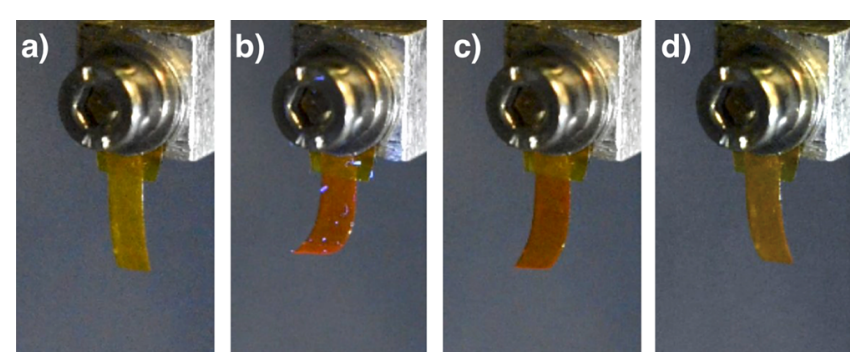

Figure 3. Photomechanical response of a free-standing rectangularshaped LCE strip (90 $\mu \mathrm{m}$ thick) with uniaxial orientation. Director along the long axis of the strip. Camera images acquired (a) before UV illumination $(t=0),(\mathrm{b})$ after $4 \mathrm{~min}$ of UV illumination $(365 \mathrm{~nm}, 50$ $\mathrm{mW} / \mathrm{cm}^{2}$ ), (c) after $2 \mathrm{~min}$ of ceasing the UV illumination, and (d) after $30 \mathrm{~min}$ of blue light illumination $\left(455 \mathrm{~nm}, 4 \mathrm{~mW} / \mathrm{cm}^{2}\right)$. Bending is produced toward the UV light source. UV light and blue light illuminate the same side of the film.

$(365 \mathrm{~nm}$ ) of one of the sides of a free-standing film (Figure 3a) having a uniaxially oriented director along its long axis, the same director orientation as that used in the thermoactuation experiments, leads to film bending toward the UV light source, as shown in Figure $3 \mathrm{~b}$. This deformation is caused by the gradient of light intensity over the sample thickness. ${ }^{44,45}$ When UV illumination ceases, the sample partially relaxes back in few seconds, retaining some remnant bending deformation, as shown in Figure 3c, with an image acquired 2 min after UV light is switched off. Subsequent irradiation of the LCE element (the same side as that irradiated with UV light) with $4 \mathrm{~mW} / \mathrm{cm}^{2}$ of blue light for $30 \mathrm{~min}$ leads to a full recovery of its initial shape (Figure 3d). The LCE element also recovers its initial shape if, instead of irradiating with blue light, it is left in darkness for $24 \mathrm{~h}$. The color of the LCE element changes along the different stages of the experiment, from yellow-orange (Figure 3a), before UV irradiation, to dark orange (Figure 3c), just after UV irradiation. As mentioned above, this photochromic behavior is associated with the transition from trans to UV-generated cis isomers, with a long lifetime at RT. ${ }^{42}$ Illumination with blue light allows the sample to recover its initial yellow-orange color again (Figure 3d) due to the light-induced isomerization of the cis state back into the trans form. Alternatively, the sample recovers this same yellow-orange color if it is left in darkness for $24 \mathrm{~h}$ due to the thermally induced isomerization of the cis isomer to the trans state. In order to gain insights about the origin of the photoinduced deformation, the surface temperature of the illuminated strip is monitored by observing the irradiated side of the LCE strip with a thermographic camera (Figure S1 of the 

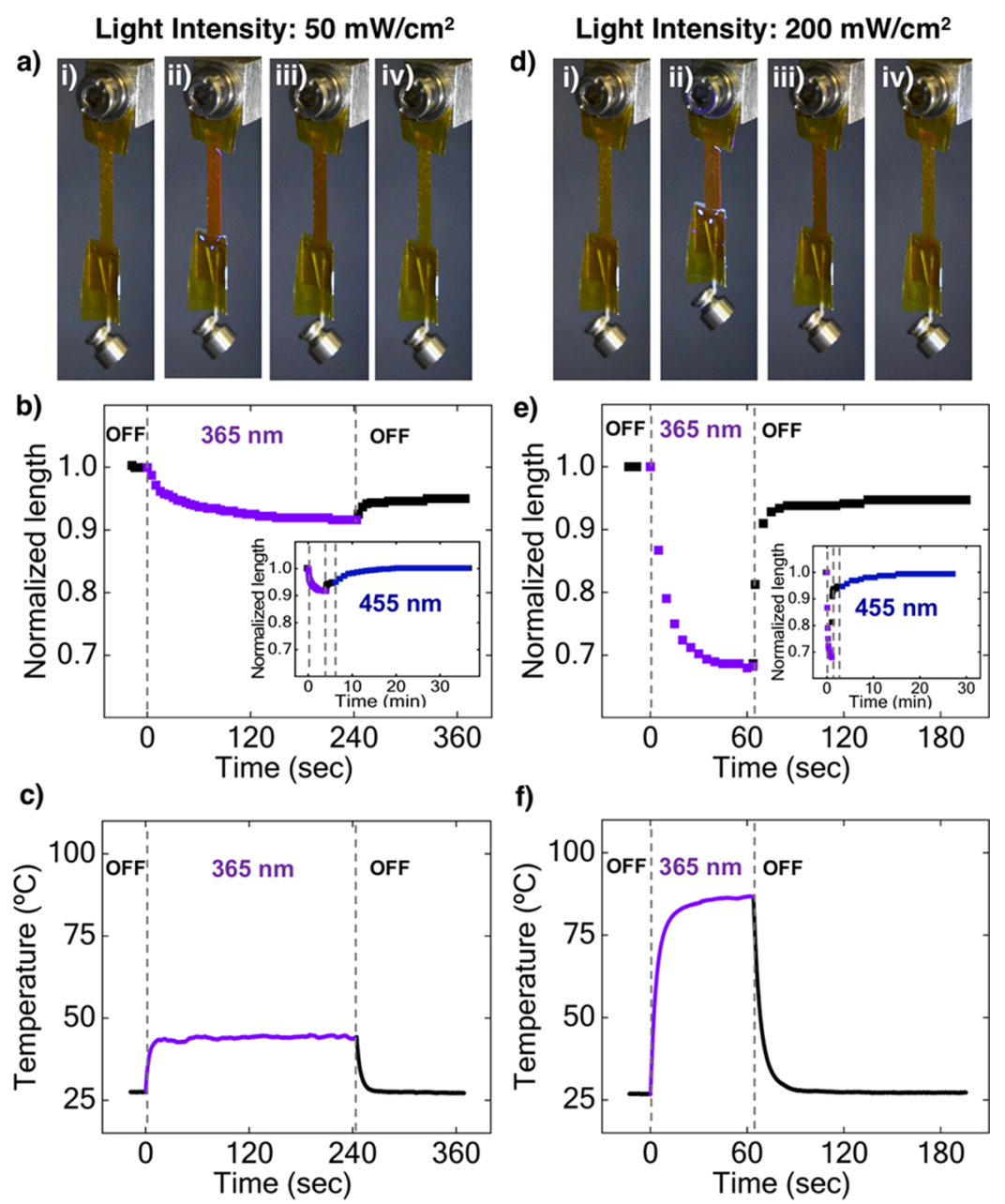

Figure 4. Photoresponse of a rectangular LCE strip ( $90 \mu \mathrm{m}$ thick) with uniaxial orientation of the director along the long axis of the element with (a-c) low UV intensity $\left(365 \mathrm{~nm}, 50 \mathrm{~mW} / \mathrm{cm}^{2}\right)$ and $(\mathrm{d}-\mathrm{f})$ high $\mathrm{UV}$ intensity $\left(365 \mathrm{~nm}, 200 \mathrm{~mW} / \mathrm{cm}^{2}\right)$ exposure. A weight of $1 \mathrm{~g}$ is attached to the lower extreme of the strip. (a,d) Images of the LCE element successively acquired (i) before UV illumination, (ii) after UV illumination (after 4 and 1 min of illumination with 50 and $200 \mathrm{~mW} / \mathrm{cm}^{2}$, respectively), (iii) 2 min after ceasing the UV illumination, and (iv) after 30 min of blue light illumination (455 $\left.\mathrm{nm}, 4 \mathrm{~mW} / \mathrm{cm}^{2}\right)$. (b,e) Temporal dependence of normalized strip length during the initial part of the experiment showing contraction upon UV illumination (purple squares) with (b) 50 and (e) $200 \mathrm{~mW} / \mathrm{cm}^{2}$ and the partial recovery of the length when UV illumination ceases (black squares). (b and $\mathrm{d}$ insets) Temporal dependence of normalized strip length during the whole experiment including the final blue light irradiation step (455 $\mathrm{nm}, 4$ $\mathrm{mW} / \mathrm{cm}^{2}$, for $30 \mathrm{~min}$, blue squares). (c,f) Temporal dependence of temperature of the irradiated side of the LCE element illuminated (365 nm, purple line) with (c) 50 and (f) $200 \mathrm{~mW} / \mathrm{cm}^{2}$.

Supporting Information). At its maximum bending, just before the illumination period ends, a temperature of $45^{\circ} \mathrm{C}$ is reached at the irradiated surface of the sample.

The observed bending behavior in our 4D printed uniaxially oriented LCE elements has also been previously observed in azocontaining LC cross-linked systems having this same director profile but obtained using other methodologies, such as rubbing of alignment layers in thin LC cells. ${ }^{44,45}$ Irradiation of the LCE element with UV generates a population of cis isomers, leading to the dark orange color of the sample. ${ }^{42}$ This population is expected to be larger at the irradiated side due to the large extinction coefficient of azobenzene chromophores that attenuates incoming UV light as it penetrates through the azocontaining element. ${ }^{27,41,44-46}$ Besides, gradients in temperature across the film thickness could also be expected due to larger heating of the sample at the illuminated side of the film. A difference of $1{ }^{\circ} \mathrm{C}$ has been estimated between the two sides of the film at the end of the UV irradiation step when equilibrium is reached but might be larger at the onset of irradiation. Both photochemical and photothermal effects contribute to the larger contraction of the illuminated side of the LCE element along the director and therefore induce film bending to accommodate the generated stresses, as observed in Figure $3 \mathrm{~b}$. When UV light is switched off, the temperature quickly relaxes to RT in few seconds, and the thermal gradient disappears that could account for the partial relaxation of the bending deformation, as observed in Figure 3c. At this point of the experiment, the population of the long-lived cis isomers remains, as supported by the dark orange color of the film, leading to the remnant bending deformation. Either by illuminating with blue light or by leaving the film in darkness for $24 \mathrm{~h}$, the azobenzene chromophores in the cis state transform back into the thermodynamically stable trans form, as indicated by the yellow-orange color of the film at the end of the experiment. As a result, the film essentially recovers the initial shape (Figure $3 \mathrm{~d}$ ).

Our focus in this manuscript is to investigate the ability of this system to perform mechanical work and exert forces. Therefore, first, we explored the lifting capacity of these 4D printed elements to raise a fixed weight, when illuminated with UV light of different intensities, namely, $50 \mathrm{~mW} / \mathrm{cm}^{2}$ (Figure $4 \mathrm{a}-\mathrm{c}$ and 
Movie S1 of the Supporting Information) and $200 \mathrm{~mW} / \mathrm{cm}^{2}$ (Figure $4 \mathrm{~d}-\mathrm{f}$ and Movie S2 of the Supporting Information). An LCE strip was studied, in a similar configuration as in the thermoactuation experiments (Figure 2), by fixing its upper extreme and attaching $1 \mathrm{~g}$ of weight at its lower one. Illumination of the strip with $50 \mathrm{~mW} / \mathrm{cm}^{2}$ of UV light $(365 \mathrm{~nm})$ induces, in this case, no bending of the strip as the weight prevents this deformation mode along the whole experiment. This lack of bending enables us to precisely quantify the dimensions of the LCE strip as a function of time by image analysis (Figure $4 b$ ). Images were extracted from video files recorded with 60 frames per second and $1920 \times 1080$ pixels. Images were then stacked into Movie S1 of the Supporting Information. By comparing the length of the element before UV irradiation (Figure 4a-i) and after a UV irradiation period of $240 \mathrm{~s}$ (Figure 4a-ii), a contraction of $8 \%$ along the director has been determined (Figure 4b). Cease of illumination resulted in a fast relaxation of the LCE strip in few seconds to a new equilibrium length that is $5 \%$ of the initial length, as seen in Figure 4a-iii, taken 2 min after illumination with UV light has been stopped (Figure 4b). Subsequent illumination of the LCE element with $4 \mathrm{~mW} / \mathrm{cm}^{2}$ of blue light $(455 \mathrm{~nm})$ for 30 min makes the sample fully recover its initial length with a margin of error of $1 \%$ (Figure $4 a-i v$ and Figure 4b). Similar changes in the color of the sample, to those identified in the photoinduced bending experiments, have been observed. Also, heating of the LCE element is induced by UV light reaching a temperature of $44^{\circ} \mathrm{C}$ at the irradiated surface of the sample at the moment of its maximum contraction, just before the illumination period ends, as shown in Figure 4c. Weight-lifting thermoactuation experiments with the LCE strip carrying the same load (Figure 2) demonstrated that heating to this same temperature led to a contraction of approximately $3 \%$, below the $8 \%$ attained with UV light illumination of the LCE element under the same load.

Focusing on the dynamics of the light-induced contraction (Figure 4b) and trying to correlate with the temperature measurement (Figure 4c) during UV irradiation, the fast increase in the temperature, reaching $90 \%$ of its final value in approximately $10 \mathrm{~s}$, is in contrast with an overall slower buildup of the contraction. Cease of the UV illumination resulted in a fast decrease in temperature (90\% drop of temperature in $13 \mathrm{~s}$ ) and, as mentioned above, an also initial rapid relaxation of the LCE strip contraction in few seconds reaching a remnant contraction of 5\%, 2 min after UV light was switched off. After 30 min of blue light illumination, the sample has essentially recovered its initial length (see the inset of Figure 4b). Repetition of the experiment under the same irradiation conditions leads to a reproducible mechanical deformation within $\pm 5 \%$.

Irradiation of the sample at higher intensities has also been explored (Figure $4 \mathrm{~d}-\mathrm{f}$ ). The same sample, which was exposed to $50 \mathrm{~mW} / \mathrm{cm}^{2}$, was used after the 30 min illumination period with blue light to ensure complete relaxation of the cis isomers to relax back to the trans state. The LCE element, shown in Figure 4d-i, is exposed in this experiment to UV light $(365 \mathrm{~nm})$ with an intensity of $200 \mathrm{~mW} / \mathrm{cm}^{2}$, four times larger than in the previous lower intensity experiment (Figure $4 a-c$ ), and with an irradiation time four times shorter $(60 \mathrm{~s})$, in order to provide the same total light dose to the sample in both experiments. Excitation of the sample with this intensity induces, after $60 \mathrm{~s}$, a contraction along the director and the weight lifting over a distance of $4.8 \mathrm{~mm}$ as shown in Figure 4d-ii. At the maximum contraction state, with $32 \%$ shortening with respect to the initial length, temperatures up to $87^{\circ} \mathrm{C}$ are reached at the surface of the sample.

A fast increase in the temperature reaching $90 \%$ of its maximum value in less than $15 \mathrm{~s}$ is observed upon exposure to $200 \mathrm{~mW} / \mathrm{cm}^{2} \mathrm{UV}$ light (Figure $4 \mathrm{f}$ ), qualitatively similar to that observed for the lower light intensity experiment (Figure 4c). Differently, the buildup of the contraction in Figure $4 \mathrm{e}$ follows a faster dynamics in this high intensity experiment when compared with that of the lower one in Figure 4b. Similar to the low intensity experiment, when UV light is switched off for the high light intensity experiment, a fast decrease in temperature (90\% drop of temperature in $12 \mathrm{~s}$ ) and a fast initial relaxation of the contraction are observed in few seconds reaching a remnant 5\% strip length shorter than the initial one.

The observed phenomena in the weight-lifting experiments suggest that photothermal and photochemical contributions to the strip contraction need to be considered. Regarding the photothermal effects that are shown in Figure 4f, temperatures as high as $87{ }^{\circ} \mathrm{C}$ were reached upon UV illumination with high power $\left(200 \mathrm{~mW} / \mathrm{cm}^{2}\right)$. In weight-lifting thermoactuation experiments (Figure 2), at $87^{\circ} \mathrm{C}$, the LCE strip, carrying the same load, contracted less than $23 \%$ by heating, which is below the $32 \%$ contraction that was reached by exposure to high intensity UV light. The large temperatures achieved upon high intensity UV light irradiation indicate that photoinduced heating of the sample has to be a relevant mechanism contributing to the observed deformation at this high intensity illumination regime. The observed fast partial relaxation when UV light is switched off can be correlated to the fast temperature relaxation (Figure $4 \mathrm{f}$ ). However, the remnant deformation after switching off UV light cannot be explained by the photothermal contribution, and further mechanisms need to be considered. Besides the photothermal effects, the changes of the scalar order parameter produced by the presence of photoinduced bulky cis isomers have also a relevant contribution to the contraction. As mentioned above, the cis isomer of the employed azobenzene chromophore, with oxygen atoms in the para positions, presents a long lifetime of several hours at RT that accounts for the remnant deformation observed after UV light is switched off while the temperature is quickly relaxed to RT (Figure 4e,f). As a further confirmation of this photochemical contribution, subsequent irradiation of this sample with blue light is performed. As a result, return of the bulky cis isomers back to the pro-mesogenic trans isomers takes place, leading to complete recovery of the initial length of the $4 \mathrm{D}$ printed LCE element (Figure $4 \mathrm{e}$ inset, blue line). It is noted that with blue light irradiation, under the applied conditions, the relaxation is in the order of tens of minutes. Faster relaxation might be easily achieved by increasing the applied blue light intensity. Dynamics might also be different for films with different thicknesses due to light attenuation dictated by Beer's law and could be optimized by changing to thinner printed elements.

In the case of the low intensity experiments with $50 \mathrm{~mW} / \mathrm{cm}^{2}$, lower temperatures $\left(44^{\circ} \mathrm{C}\right.$, as mentioned above) are reached during UV irradiation, when compared to the high intensity experiment; so, the photothermal contribution is also smaller. Although there is an interplay between photothermal and photochemical effects due to the changes of absorption spectra induced by light and the temperature-dependent trans to cis and cis to trans isomerization rates, a similar remnant contraction of $5 \%$ has been reached in both high and low intensity experiments (same total dose), which tentatively could be ascribed to a 
similar cis population reached after UV illumination and temperature relaxation processes for both intensity regimes.

By characterizing the capacity to raise a fixed weight with exposure to UV light, we can gain information on the ability of our system to perform mechanical work. The active part of the strip, with an estimated weight of $4 \mathrm{mg}$, lifted a total weight of $1.02 \mathrm{~g}$, including the adapter connecting the strip and the $1 \mathrm{~g}$ load. As a result, a weight-specific work capacity of $12 \mathrm{~J} / \mathrm{kg}$ is reached for $200 \mathrm{~mW} / \mathrm{cm}^{2}$ light intensity, which is 4 times higher than that reached by $50 \mathrm{~mW} / \mathrm{cm}^{2}(3 \mathrm{~J} / \mathrm{kg})$. Larger values have been reported in the literature for the work capacity of $4 \mathrm{D}$ printed thermoresponsive LC cross-linked systems. ${ }^{20}$ Although the comparison is not straightforward with thermoactuation, in the present case with light as a stimulus, we might not be taking full advantage of the material actuation potential due to light attenuation in the film thickness as dictated by Beer's law. Further material optimization with respect to azobenzene chromophore content, and therefore light penetration, as well as regarding polymer architecture or element thickness could also be done to further exploit the capabilities of this printing platform to generate high work capacity actuators. Advantageously, the ability of our 4D printing platform to generate complex director patterns will give access to other modes of actuation, beyond muscle-like linear actuators, such as the cones theoretically predicted by Warner et al. ${ }^{47}$ and experimentally implemented by others. ${ }^{38,48,49}$

To further explore the relevance of these $4 \mathrm{D}$ printed elements as photomechanical actuators, the photomechanical response to light has been characterized by performing isometric force experiments (Figure 5). These were carried out in dog bone-
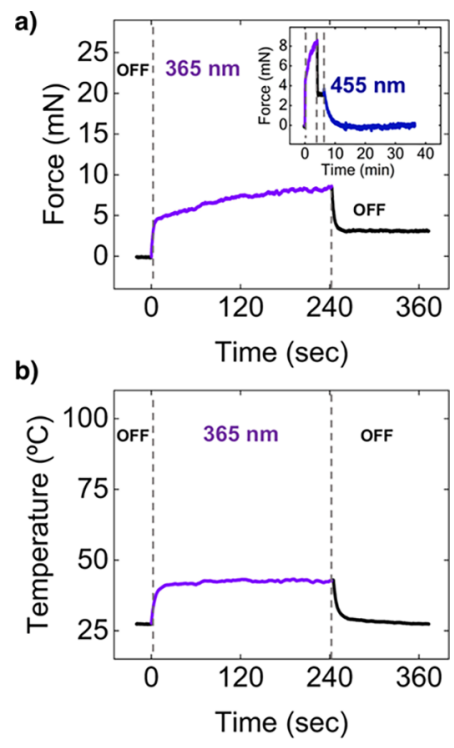

Figure 5. Temporal dependence of (a) photoinduced force and (b) temperature under exposure to low UV light intensity $\left(50 \mathrm{~mW} / \mathrm{cm}^{2}\right)$ carried out with a dog bone-shaped LCE strip (110 $\mu \mathrm{m}$ thick, sample cross-sectional area of $0.31 \mathrm{~mm}^{2}$ ) with uniaxial orientation of the director along the long axis of the element. A prestretching of the sample by a force of $3.1 \mathrm{mN}$ has been applied prior to force measurement. The sample is illuminated with UV light $(365 \mathrm{~nm})$ for $240 \mathrm{~s}$ (illumination starts at $t=0 \mathrm{~s}$, purple line), and UV light is switched off; the sample is left to relax without light for $120 \mathrm{~s}$ (black line). (a inset) Photoinduced force measured after this relaxation period while the LCE strip is illuminated with $4 \mathrm{~mW} / \mathrm{cm}^{2}$ of blue light $(455 \mathrm{~nm})$ for $30 \mathrm{~min}$. shaped LCE strips (110 $\mu \mathrm{m}$ thick) with the same uniaxial orientation as in the previous weight-lifting experiments. One extreme of the sample is clamped to a force transducer and the other to a translation stage that allows us to generate, before photoexposure, a prestretching of the sample by a force of 3.1 $\mathrm{mN}$ (sample cross-sectional area of $0.31 \mathrm{~mm}^{2}$ ).

Similar to those for the weight-lifting experiments, irradiation of an LCE strip with a sequential illumination scheme (first UV and later blue light) is carried out. We registered the photoinduced force as a function of time (Figure 5a) and surface temperature at the illuminated side (Figure 5b). Upon irradiation with UV light, a fast increase in temperature is measured from Figure $5 b$, while force builds up over the illumination period at a slower pace than temperature as shown in Figure 5a. Cease of UV light irradiation leads to a fast relaxation of the temperature accompanied by a fast partial force relaxation, leaving a remnant stress (36\% of the peak force). Later, irradiation with blue light leads to a sudden small increase in force, which could be ascribed to a $3{ }^{\circ} \mathrm{C}$ photoinduced heating observed when blue light reaches the LCE element, followed by a slow relaxation of the force to essentially the initial value (inset of Figure 5a, blue line). Overall, the experimental results obtained are consistent with weight-lifting experiments and can be explained considering the same photothermal and photochemical mechanisms.

Irradiation of the sample with UV light pulses of $1 \mathrm{~s}$ at different light intensities leads to a rapid generation of forces as shown in Figure 6. The peak force is identified to be light powerdependent, and larger forces are obtained for higher light intensities (Figure 6a, c, and e). For these short UV light pulses, when illumination ceases, forces relax back essentially to their initial values, with no measurable remnant force (within the experimental error). The force relaxation was fitted to an exponential decay, and relaxation times of around 2-3 s were extracted.

In order to provide a sense of the repeatability in these isometric force experiments with pulsed excitation, a sequence of pulses (pulse duration of $1 \mathrm{~s}$ with a periodicity of $11 \mathrm{~s}$ ) has been supplied to the sample, finding reproducible values of the peak force within $\pm 5 \%$ (Figure S2 of the Supporting Information). It is important to note that when a large sequence of pulses is applied on the sample, this leads to a remnant force in a qualitatively equivalent fashion as the same dose that continuous UV illumination produces. This could be ascribed to the population of cis isomers on accumulation of light dose. In addition, thermographic measurements (Figure $6 \mathrm{~b}, \mathrm{~d}$, and $\mathrm{f}$ ) enable us to determine the peak temperature reached at the illuminated side of the sample. Temperatures of 30, 33, and 38 ${ }^{\circ} \mathrm{C}$ are measured at the end of the $1 \mathrm{~s}$ exposure period for excitation energies of 50,100 , and $200 \mathrm{~mW} / \mathrm{cm}^{2}$, respectively. After light is switched off, the temperature goes back to RT also in few seconds.

The obtained experimental results, with fast buildup and relaxation dynamics and a nonmeasurable remnant force after one pulse, indicate that the photothermal effect is dominant in the short single-pulse scheme. LCE elements, when triggered with a short-pulsed light stimulus of enough intensity, quickly transform light energy into a significant amount of heat and therefore generate measurable mechanical forces through a photothermal effect. The provided light dose is not enough to generate a sufficient cis population to lead to measurable remnant photochemical actuation, although repeated activation would populate the cis state. 
$50 \mathrm{~mW} / \mathrm{cm}^{2}$

a)
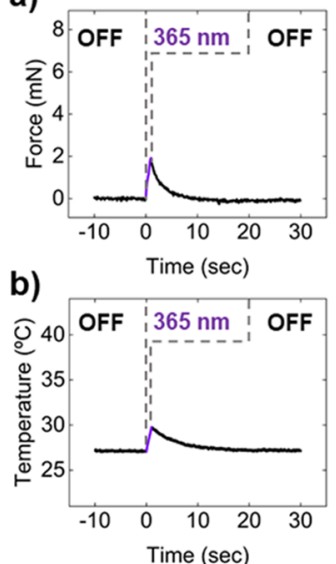

$100 \mathrm{~mW} / \mathrm{cm}^{2}$

c)

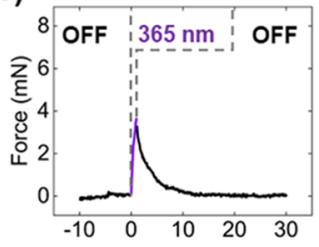

d) Time (sec)

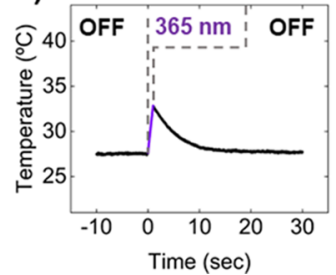

$200 \mathrm{~mW} / \mathrm{cm}^{2}$

e)

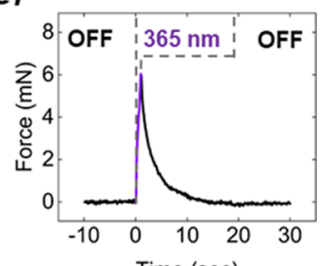

f)

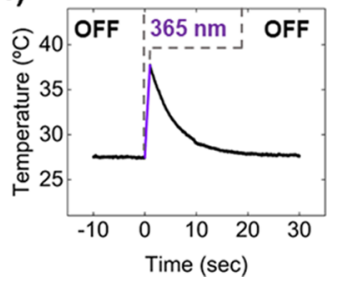

Figure 6. Photoresponse of a dog bone-shaped LCE strip ( $110 \mu \mathrm{m}$ thick) with uniaxial orientation. Director along the long axis of the element. A prestretching of the sample by a force of $2.4 \mathrm{mN}$ has been applied prior to force measurements. Temporal dependence of force measurement under pulsed excitation ( $1 \mathrm{~s}$, illumination starts at $t=0 \mathrm{~s}$ ) with UV light ( $365 \mathrm{~nm}$, purple line) at (a) 50, (c) 100, and (e) $200 \mathrm{~mW} / \mathrm{cm}^{2}$. Temporal dependence of the sample surface temperature under pulsed excitation ( $1 \mathrm{~s}$, illumination starts at $t=0 \mathrm{~s}$ ) with UV light (365 nm, purple line) at (b) 50, (d) 100, and (f) $200 \mathrm{~mW} / \mathrm{cm}^{2}$.

\section{CONCLUSIONS}

The 4D printing of azobenzene-containing LCE elements that respond to light has been reported. Digital deposition of an acrylate end-capped liquid crystalline polymer containing an azobenzene moiety and subsequently capturing both shape and morphology by photopolymerization give muscle-like elements that act upon temperature and, more importantly for this publication, light. A key component in the printing process is the printing needle that feeds the reactive ink to the substrate through microextrusion. The corresponding flow aligns the liquid crystalline polymer and thereby also the in-chain azobenzene moieties. As a result, various shapes can be written, and the director orientation in the responsive elements is set by the deposition direction. Changes of the scalar order parameter, induced either by temperature or by light, generate contraction along the director and expansion perpendicular to that, which provides the director-controlled muscle function. In a muscle configuration, the forces in the presented isometric force experiments easily reached values of $24 \mathrm{kPa}$ without further optimization. Preliminary experiments revealed that this value could be manifold by increasing the light intensity. Besides, the weight-specific work that is delivered reaches $12 \mathrm{~J} / \mathrm{kg}$. Importantly, on request, the photogenerated force can be maintained or switched off by two-wavelength photochemical means or by temperature. In analogy, the printed elements can be used for push or pull purposes when integrated in complex devices such as (soft) robots. Adjusted deformation or force can be achieved by controlled light intensities. Also, memory effects can be erased by a temperature pulse.

The mechanism of photoactuation involves photothermal and photochemical aspects that might work in conjunction or separately, depending on the actuation wavelength, light intensity, and pulse duration. The application of short pulses circumvents to a significant extent the azobenzene transition to its cis state, while the photothermal effect dominates, leading to no measurable remnant deformation after the pulse. This enables repetitive muscle actuation of interest for driving (micro)machines and robot-integrated elements. Related to the materials and processing, the elements overcome the problem of brittleness often observed for thin-film liquid crystal networks made from single-entity liquid crystal monomers.

Beyond the demonstrated linear photoactuators, which could have been achieved by other processing methods, the 4D printing platform presented in this work allows us to progress toward the implementation of elements with digitally defined director fields. We are currently working in this direction in search of novel photodriven soft robotic elements and devices.

\section{ASSOCIATED CONTENT}

\section{Supporting Information}

The Supporting Information is available free of charge at https://pubs.acs.org/doi/10.1021/acsami.0c13341.

(Figure S1) Thermographic image of a free-standing rectangular-shaped LCE strip $(90 \mu \mathrm{m}$ thick $)$ with uniaxial orientation and $\left(50 \mathrm{~mW} / \mathrm{cm}^{2}\right.$ illumination) (Figure S2) photoresponse of a dog bone-shaped LCE strip with uniaxial orientation under a sequence of ten pulses (50 $\mathrm{mW} / \mathrm{cm}^{2}$ illumination) (PDF)

(Movie S1) Light actuation of a rectangular LCE strip (90 $\mu \mathrm{m}$ thick) with uniaxial orientation $\left(50 \mathrm{~mW} / \mathrm{cm}^{2}\right.$ illumination) (AVI)

(Movie S2) Light actuation of a rectangular LCE strip (90 $\mu \mathrm{m}$ thick) with uniaxial orientation $\left(200 \mathrm{~mW} / \mathrm{cm}^{2}\right.$ illumination) (AVI)

\section{AUTHOR INFORMATION}

\section{Corresponding Author}

Carlos Sánchez-Somolinos - Instituto de Nanociencia y Materiales de Aragón (INMA), CSIC-Universidad de Zaragoza, Departamento de Física de la Materia Condensada, Zaragoza 50009, Spain; CIBER in Bioengineering, Biomaterials and Nanomedicine (CIBERBBN), 28029 Madrid, Spain; ○ orcid.org/0000-0003-3900-2866; Email: carlos.s@csic.es

\section{Authors}

Lorena Ceamanos - Instituto de Nanociencia y Materiales de Aragón (INMA), CSIC-Universidad de Zaragoza, 
Departamento de Física de la Materia Condensada, Zaragoza 50009, Spain

Zehra Kahveci - Instituto de Nanociencia y Materiales de Aragón (INMA), CSIC-Universidad de Zaragoza, Departamento de Física de la Materia Condensada, Zaragoza 50009, Spain

María López-Valdeolivas - Instituto de Nanociencia y Materiales de Aragón (INMA), CSIC-Universidad de Zaragoza, Departamento de Física de la Materia Condensada, Zaragoza 50009, Spain

Danqing Liu - Stimuli-Responsive Functional Materials and Devices Group, Department of Chemical Engineering and Chemistry and Institute of Complex Molecular Systems, Eindhoven University of Technology, 5600 MB Eindhoven, The Netherlands; (ㅇ) orcid.org/0000-0001-8830-0443

Dirk Jan Broer - Stimuli-Responsive Functional Materials and Devices Group, Department of Chemical Engineering and Chemistry and Institute of Complex Molecular Systems, Eindhoven University of Technology, 5600 MB Eindhoven, The Netherlands; 10 orcid.org/0000-0001-6136-3276

Complete contact information is available at: https://pubs.acs.org/10.1021/acsami.0c13341

\section{Author Contributions}

C.S.-S., D.J.B., and D.L. conceived the materials and research. L.C. and C.S.-S. set up the methodology, L.C., M.L.-V., and Z.K. performed the experiments and analyzed the experimental results. All authors discussed the experimental results. Z.K., L.C., and C.S.-S. composed the first draft. All authors reviewed and improved the initial draft and approved the final manuscript.

\section{Funding}

The described research study is part of the project PRIME. This project has received funding from the European Union's Horizon 2020 Research and Innovation Programme under grant agreement no. 829010 (PRIME). Funding has also been received from the Spanish MINECO project BIO2017-84246C2-1-R, Gobierno de Aragón project LMP150_18, and FEDER (EU). D.J.B. thanks the European Research Commission (ERC) Advanced Grant 66999 (VIBRATE). D.L. thanks the Netherlands Organization of Scientific Research (NWO) VENI grant 15135.

\section{Notes}

The authors declare no competing financial interest.

\section{ACKNOWLEDGMENTS}

The authors thank Chris van Heesch for conceptual illustrations in Figure $1 b$.

\section{REFERENCES}

(1) Ligon, S. C.; Liska, R.; Stampfl, J.; Gurr, M.; Mülhaupt, R. Polymers for $3 \mathrm{D}$ Printing and Customized Additive Manufacturing. Chem. Rev. 2017, 117, 10212-10290.

(2) Tofail, S. A. M.; Koumoulos, E. P.; Bandyopadhyay, A.; Bose, S.; O'Donoghue, L.; Charitidis, C. Additive Manufacturing: Scientific and Technological Challenges, Market Uptake and Opportunities. Mater. Today 2018, 21, 22-37.

(3) Rus, D.; Tolley, M. T. Design, Fabrication and Control of Soft Robots. Nature 2015, 521, 467-475.

(4) Huang, S. H.; Liu, P.; Mokasdar, A.; Hou, L. Additive Manufacturing and its Societal Impact: A Literature Review. Int. J. Adv. Manuf. Technol. 2013, 67, 1191-1203.

(5) Guo, N.; Leu, M. C. Additive Manufacturing: Technology, Applications and Research Needs. Front. Mech. Eng. 2013, 8, 215-243.
(6) Tibbits, S. The emergence of " $4 D$ printing". https://www.ted.com/ talks/skylar_tibbits_the_emergence_of_4d_printing (accessed April, 2020).

(7) Tibbits, S. 4D Printing: Multi-Material Shape Change. Archit. Des. 2014, 84, 116-121.

(8) Huang, L.; Jiang, R.; Wu, J.; Song, J.; Bai, H.; Li, B.; Zhao, Q.; Xie, T. Ultrafast Digital Printing Toward 4D Shape Changing Materials. Adv. Mater. 2017, 29, 1605390.

(9) Wu, J.-J.; Huang, L.-M.; Zhao, Q.; Xie, T. 4D Printing: History and Recent Progress. Chin. J. Polym. Sci. 2018, 36, 563-575.

(10) De Marco, C.; Pané, S.; Nelson, B. J. 4D Printing and Robotics. Sci. Rob. 2018, 3, No. eaau0449.

(11) del Barrio, J.; Sánchez-Somolinos, C. Light to Shape the Future: From Photolithography to 4D Printing. Adv. Optical Mater. 2019, 7, 1900598.

(12) Zarek, M.; Layani, M.; Cooperstein, I.; Sachyani, E.; Cohn, D.; Magdassi, S. 3D Printing of Shape Memory Polymers for Flexible Electronic Devices. Adv. Mater. 2016, 28, 4449-4454.

(13) Yang, H.; Leow, W. R.; Wang, T.; Wang, J.; Yu, J.; He, K.; Qi, D.; Wan, C.; Chen, X. 3D Printed Photoresponsive Devices Based on Shape Memory Composites. Adv. Mater. 2017, 29, 1701627.

(14) Wei, H.; Zhang, Q.; Yao, Y.; Liu, L.; Liu, Y.; Leng, J. Direct-Write Fabrication of 4D Active Shape-Changing Structures Based on a Shape Memory Polymer and its Nanocomposite. ACS Appl. Mater. Interfaces 2017, 9, 876-883.

(15) Jin, B.; Song, H.; Jiang, R.; Song, J.; Zhao, Q.; Xie, T. Programming a Crystalline Shape Memory Polymer Network with Thermo- and Photo-Reversible Bonds Toward a Single-Component Soft Robot. Sci. Adv. 2018, 4, No. eaao3865.

(16) Sydney Gladman, A.; Matsumoto, E. A.; Nuzzo, R. G.; Mahadevan, L.; Lewis, J. A. Biomimetic 4D Printing. Nat. Mater. 2016, 15, 413-418.

(17) Kim, J.; Hanna, J. A.; Byun, M.; Santangelo, C. D.; Hayward, R C. Designing Responsive Buckled Surfaces by Halftone Gel Lithography. Science 2012, 335, 1201-1205.

(18) Ambulo, C. P.; Burroughs, J. J.; Boothby, J. M.; Kim, H.; Shankar, M. R.; Ware, T. H. Four-Dimensional Printing of Liquid Crystal Elastomers. ACS Appl. Mater. Interfaces 2017, 9, 37332-37339.

(19) López-Valdeolivas, M.; Liu, D.; Broer, D. J.; Sánchez-Somolinos, C. 4D Printed Actuators with Soft-Robotic Functions. Macromol. Rapid Commun. 2018, 39, 1700710.

(20) Kotikian, A.; Truby, R. L.; Boley, J. W.; White, T. J.; Lewis, J. A. 3D Printing of Liquid Crystal Elastomeric Actuators with Spatially Programed Nematic Order. Adv. Mater. 2018, 30, 1706164.

(21) Broer, D. J.; Mol, G. N. Anisotropic Thermal Expansion of Densely Cross-linked Oriented Polymer Networks. Polym. Eng. Sci. 1991, 31, 625-631.

(22) Saed, M. O.; Ambulo, C. P.; Kim, H.; De, R.; Raval, V.; Searles, K.; Siddiqui, D. A.; Cue, J. M. O.; Stefan, M. C.; Shankar, M. R.; Ware, T. H. Molecularly-Engineered, 4D-Printed Liquid Crystal Elastomer Actuators. Adv. Funct. Mater. 2019, 29, 1806412.

(23) Barnes, M.; Sajadi, S. M.; Parekh, S.; Rahman, M. M.; Ajayan, P. M.; Verduzco, R. Reactive 3D Printing of Shape-Programmable Liquid Crystal Elastomer Actuators. Appl. Mater. Interfaces 2020, 12, 2869228699.

(24) Kotikian, A.; McMahan, C.; Davidson, E. C.; Muhammad, J. M.; Weeks, R. D.; Daraio, C.; Lewis, J. A. Untethered Soft Robotic Matter with Passive Control of Shape Morphing and Propulsion. Sci. Robotics 2019, 4, No. eaax7044.

(25) Yu, Y.; Nakano, M.; Ikeda, T. Directed Bending of a Polymer Film by Light. Nature 2003, 425, 145.

(26) Yamada, M.; Kondo, M.; Mamiya, J.-i.; Yu, Y.; Kinoshita, M.; Barrett, C. J.; Ikeda, T. Photomobile Polymer Materials: Towards Light-Driven Plastic Motors. Angew. Chem., Int. Ed. 2008, 47, 49864988.

(27) White, T. J.; Broer, D. J. Programmable and Adaptive Mechanics with Liquid Crystal Polymer Networks and Elastomers. Nat. Mater. 2015, 14, 1087-1098. 
(28) van Oosten, C. L.; Bastiaansen, C. W. M.; Broer, D. J. Printed Artificial Cilia from Liquid-Crystal Network Actuators Modularly Driven by Light. Nat. Mater. 2009, 8, 677-682.

(29) Gelebart, A. H.; Mc Bride, M.; Schenning, A. P. H. J.; Bowman, C. N.; Broer, D. J. Photoresponsive Fiber Array: Toward Mimicking the Collective Motion of Cilia for Transport Applications. Adv. Funct. Mater. 2016, 26, 5322-5327.

(30) Iamsaard, S.; Aßhoff, S. J.; Matt, B.; Kudernac, T.; Cornelissen, J. J. L. M.; Fletcher, S. P.; Katsonis, N. Conversion of Light into Macroscopic Helical Motion. Nat. Chem. 2014, 6, 229-235.

(31) Gelebart, A. H.; Jan Mulder, D.; Varga, M.; Konya, A.; Vantomme, G.; Meijer, E. W.; Selinger, R. L. B.; Broer, D. J. Making Waves in a Photoactive Polymer Film. Nature 2017, 546, 632-636.

(32) Koerner, H.; White, T. J.; Tabiryan, N. V.; Bunning, T. J.; Vaia, R. A. Photogenerating Work from Polymers. Mater. Today 2008, 11, 3442.

(33) Camacho-Lopez, M.; Finkelmann, H.; Palffy-Muhoray, P.; Shelley, M. Fast Liquid-Crystal Elastomer Swims into the Dark. Nat. Mater. 2004, 3, 307-310.

(34) Palagi, S.; Mark, A. G.; Reigh, S. Y.; Melde, K.; Qiu, T.; Zeng, H.; Parmeggiani, C.; Martella, D.; Sanchez-Castillo, A.; Kapernaum, N.; Giesselmann, F.; Wiersma, D. S.; Lauga, E.; Fischer, P. Structured Light Enables Biomimetic Swimming and Versatile Locomotion of Photoresponsive Soft Microrobots. Nat. Mater. 2016, 15, 647-653.

(35) Lv, J.; Liu, Y.; Wei, J.; Chen, E.; Qin, L.; Yu, Y. Photocontrol of Fluid Slugs in Liquid Crystal Polymer Microactuators. Nature 2016, 537, 179-184.

(36) Wani, O. M.; Zeng, H.; Priimagi, A. A Light-Driven Artificial Flytrap. Nat. Commun. 2017, 8, 15546.

(37) Ahn, S.; Ware, T. H.; Lee, K. M.; Tondiglia, V. P.; White, T. J. Photoinduced Topographical Feature Development in Blueprinted Azobenzene-Functionalized Liquid Crystalline Elastomers. Adv. Funct. Mater. 2016, 26, 5819-5826.

(38) De Haan, L. T.; Sánchez-Somolinos, C.; Bastiaansen, C. M. W.; Schenning, A. P. H. J.; Broer, D. J. Engineering of Complex Order and the Macroscopic Deformation of Liquid Crystal Polymer Networks. Angew. Chem. 2012, 51, 12469-12472.

(39) Zeng, H.; Wasylczyk, P.; Wiersma, D. S.; Priimagi, A. Light Robots: Bridging the Gap between Microrobotics and Photomechanics in Soft Materials. Adv. Mater. 2018, 30, 1703554.

(40) Warner, M. Topographic Mechanics and Applications of Liquid Crystalline Solids. Annu. Rev. Condens. Matter Phys. 2020, 11, 125-145.

(41) Ikeda, T.; Mamiya, J.; Yu, Y. Photomechanics of LiquidCrystalline Elastomers and Other Polymers. Angew. Chem., Int. Ed. 2007, 46, 506-528.

(42) Liu, D.; Broer, D. J. New Insights into Photoactivated Volume Generation Boost Surface Morphing in Liquid Crystal Coatings. Nat. Commun. 2015, 6, 8334.

(43) Kumar, G. S.; Neckers, D. C. Photochemistry of AzobenzeneContaining Polymers. 1989, 89, 1915-1925.

(44) Kondo, M.; Yu, Y.; Ikeda, T. How does the Initial Alignment of Mesogens Affect the Photoinduced Bending Behavior of LiquidCrystalline Elastomers? Angew. Chem., Int. Ed. 2006, 45, 1378-1382.

(45) Harris, K. D.; Cuypers, R.; Scheibe, P.; Van Oosten, C. L.; Bastiaansen, C. W. M.; Lub, J.; Broer, D. J. Large Amplitude LightInduced Motion in High Elastic Modulus Polymer Actuators. J. Mater. Chem. 2005, 15, 5043-5048.

(46) Cervera-Procas, R.; Sánchez-Somolinos, C.; Serrano, J. L.; Omenat, A. A Polymer Network Prepared by the Thiol-Yne Photocrosslinking of a Liquid Crystalline Dendrimer. Macromol. Rapid Commun. 2013, 34, 498-503.

(47) Modes, C. D.; Bhattacharya, K.; Warner, M. DisclinationMediated Thermo-Optical Response in Nematic Glass Sheets. Phys. Rev. E Stat. Nonlinear Soft Matter Phys. 2010, 81, No. 060701.

(48) Ware, T. H.; McConney, M. E.; Wie, J. J.; Tondiglia, V. P.; White, T. J. Voxelated Liquid Crystal Elastomers. Science 2015, 347, 982-984.

(49) Guin, T.; Settle, M. J.; Kowalski, B. A.; Auguste, A. D.; Beblo, R. V.; Reich, G. W.; White, T. J. Layered Liquid Crystal Elastomer Actuators. Nat. Commun. 2018, 9, 2531. 